\title{
Repercusión altmétrica y tipo de acceso en artículos peruanos de Ciencias Sociales
}

Joel Alhuay-Quispe*

Lourdes Bautista-Ynofuente**

Artículo recibido:

7 de mayo de 2021

Artículo aceptado:

31 de agosto de 2021

Artículo de investigación

\section{Resumen}

El artículo analiza el impacto en medios sociales de artículos peruanos del área temática de las Ciencias Sociales a través de la repercusión mediática en fuentes altmétricas y el tipo de acceso en el que se publican. Se analizan 1186 artículos con filiación de instituciones peruanas recuperados de la base de datos Scopus (2011-2020), para lo cual se emplearon seis (6) indicadores altmétricos de Altmetric y citas de Dimensions. El método de análisis compara la repercusión altmétrica en relación con el tipo de acceso: abierto o restringido, en cinco áreas temáticas de las Ciencias Sociales: Artes y Humanidades, Negocios

* $\quad$ Facultad de Ingeniería, Universidad Privada San Juan Bautista, Perú

joel.alhuay@upsjb.edu.pe

** Universidad Nacional Mayor de San Marcos, Perú

lourdes.bautista1@unmsm.edu.pe

INVESTIGACIÓN BIBLIOTECOLÓGICA, vol. 35, núm. 89, octubre/diciembre, 2021, México, ISSN: 2448-8321 pp. 91-110 
y Contabilidad, Ciencias de la Decisión, Economía y Finanzas, y Psicología. Los resultados muestran una alta correlación entre lecturas en Mendeley y citas de Dimensions $(r=0.797)$, y que existe una diferencia significativa por el tipo de acceso a la publicación en las menciones provenientes de Facebook $(p=.000)$ y Twitter $(p=.005)$. El estudio concluye que existe una mayor tendencia por parte de los autores peruanos que abarcan temas de Psicología en publicar sus artículos en acceso abierto, otorgándoles mayor visibilidad en Mendeley y Dimensions.

Palabras clave: Producción Científica; Indicadores Altmétricos; Acceso Abierto; Ciencias Sociales

\author{
Altmetric attention and access type in Peruvian ar- \\ ticles of Social Sciences \\ Joel Albuay-Quispe and Lourdes Bautista-Ynofuente
}

\begin{abstract}
This article aims to identify the impact in altmetric sources and type of publishing access in Social Sciences articles. It analyses 1,186 articles affiliated to Peruvian institutions retrieved from Scopus (2011 to 2020) using six altmetric indicators from Altmetric and Dimensions citations. The method used compares altmetric impact in relation to open or restricted access and subject areas: Arts and Humanities, Business and Accounting, Decision Sciences, Economics and Finance, and Psychology. Results show a high correlation between Mendeley reads and citations of Dimensions $(r=0.797)$, and besides there is a significant difference according to access tandype in mentions from Facebook $(p=.000)$ and Twitter $(p=.005)$. In conclusion, Peruvian Psychology authors publish mostly in some open access route and publications obtain greater visibility in Mendeley and Dimensions when they are published through green route.
\end{abstract}

Keywords: Scientific Production; Altmetric Indicators; Open Access; Social Sciences 


\section{INTRODUCCIÓN}

T a masificación de plataformas de social media ha transformado la manera Le comunicar la producción científica, así como la aparición de las denominadas métricas alternativas o altmétricas ha variado la concepción tradicional de la evaluación del impacto de las publicaciones en las Ciencias Sociales como en las demás áreas del conocimiento.

La evaluación o medición de los resultados obtenidos por un investigador y su equipo de estudio se lleva a cabo de modos distintos o complementarios: a) peer-review o evaluación por pares, y b) por valoración a posteriori de los resultados de la investigación (Peña-Rey Lorenzo, 2004).

El acceso abierto (open access en inglés) se refiere al acceso inmediato, permanente y gratuito, en línea y a texto completo de todos los artículos de revistas de investigación arbitradas (Harnad, 2005). Para Piwowar et al. (2018), el acceso abierto se define como el acceso libre para leer en línea, ya sea en el sitio web del editor o en un repositorio, por lo que todos los artículos que no cumplen esta definición son de acceso cerrado (restringido). La vía de publicación de acceso abierto (OA) surge en notoria oposición a la mercantilización del conocimiento y de la comunicación científica, posteriormente apropiado por los grandes sellos editoriales y revistas de acceso restringido (por suscripción) como modelo de negocio en proyección a su persistencia en el tiempo. El movimiento OA adquiere su naturaleza internacional a partir de tres grandes hitos: las declaraciones de Budapest (2002), Bethesda (2003), y Berlín (2003) (Harnad, 2005). En consecuencia, iniciativas supranacionales como la de la Unión Europea buscan acelerar la transición de los sistemas tradicionales de publicación cerrada hacia los de acceso abierto (Ellers, Crowther y Harvey, 2017), eliminando así el costo de suscripción para la lectura (i.e. descarga) de los contenidos. Una de las modalidades de suscripción más comunes es la institucional, la cual consiste en que una entidad realice compras de colecciones de títulos y recursos electrónicos mediante su biblioteca o quien hiciera labores de gestor de información. Por otro lado, también está la opción de acceder de manera individual mediante el pago por artículo proveniente de los recursos propios del investigador o las fuentes de financiamiento de sus proyectos.

Las revistas inicialmente publicaban sus contenidos mediante suscripción; hoy en día, se encuentran apostando por el acceso abierto o modalidad mixta. De este modo, el movimiento Open Access nace como ruta alterna de comunicación de la producción científica ante el predominio de los canales comerciales de publicación académica, permitiendo la disponibilidad del texto completo para lectura de manuscritos: la ruta verde, a través de repositorios 
digitales, y la ruta dorada, mediante las propias revistas electrónicas. La ruta dorada consiste en que los artículos se publican en acceso abierto y los costos se trasladan a los autores o entidades a las cuales se adscriben los investigadores; estos costos, denominados cargos de procesamiento o APC (article processing charges por sus siglas en inglés), se definen como las tasas que cobran los editores a los autores por la publicación de artículos de revistas (Beasley, 2016). Por otro lado, la ruta verde implica que el autor de un manuscrito publicado en una revista de acceso cerrado deposite una copia gratuita en un repositorio digital en su versión preliminar (preprint) o versión corregida (postprint). Además de estas dos categorías de publicación en acceso abierto, existen las denominadas híbrido, bronce, y la ruta cerrada. Piwowar et al. (2018) definen las cinco rutas de publicación como sigue:

- Dorada (gold): publicado en una revista de acceso abierto indexada por el DOAJ.

- Verde (green): acceso de pago en la página del editor, pero con una copia gratuita en un repositorio.

- Híbrido (bybrid): gratis bajo una licencia abierta en una revista de acceso de pago.

- Bronce (bronze): acceso libre en la página del editor, pero sin una licencia claramente identificable.

- Cerrado (closed): todos los demás artículos, incluidos los que sólo se comparten en una red privada o de acceso mediante Sci-Hub.

No obstante, a pesar de la expansión del movimiento de acceso abierto y su filosofía de abrir el conocimiento científico para todos, son los propios autores quienes no asimilan esta práctica como una vía crucial para masificar el acceso al conocimiento, además de la posibilidad de medir el impacto y reconocimiento de sus publicaciones. Este último se traduce en las citas recibidas, un indicador por excelencia para medir el impacto académico de una publicación científica; no obstante, indicadores complementarios y recientes como la repercusión en plataformas sociales y medios web podrían dar indicios tempranos de los artículos que serán posteriormente citados. Para Bornmann (2014), uno de los cambios producidos al interior de la cienciometría es el acceso abierto y el movimiento del análisis de citación en la web hacia el análisis del uso de los medios sociales, fenómeno conocido actualmente como altmetrics o altmétricas, en español. Estas métricas alternativas suponen un complemento a la evaluación de la influencia de la investigación, a partir de nuevas audiencias que van más allá de la propia comunidad académica y científica puesto que las herramientas tradicionales para el análisis bibliométrico, como 
el factor de impacto y el índice $\mathrm{H}$, no capturan el impacto de productos de investigación conectados a la web (Lahikainen, 2016). Este enfoque de complementariedad al análisis de citas tradicionales parece ser más significativo y relevante en comparación con el uso aislado de los tradicionales indicadores bibliométricos (Melero, 2015; Alonso-Arévalo, 2016; Bornmann, 2014; Costas, Zahedi y Wouters, 2015; Haustein, 2016; Wilsdon et al., 2015). Respecto a estas métricas se plantean interrogantes sobre las posibles limitaciones en el campo de la bibliometría y evaluación de la ciencia. Una de ellas es la falta de precisión respecto al objeto de medición: ¿impacto o influencia?, ¿social o científica? (Sud y Thelwall, 2014; Haustein, Bowman y Costas, 2015). Por otro lado, surgen los problemas de normalización e inconsistencia en la recuperación de los datos (Robinson-García et al., 2014) y errores de los propios autores (Alhuay-Quispe y Bautista-Ynofuente, 2020), sumado a las diferentes coberturas de las plataformas que proporcionan los datos altmétricos.

La mayoría de los estudios bibliométricos en torno a la producción científica peruana se centra en las áreas de la salud como la medicina y la odontología, mientras que sólo algunos se enfocan en las ciencias sociales como la psicología (Carranza Esteban et al., 2020; Livia, 2008) y otros analizan casos de instituciones específicas (Delgado Arenas, Delgado Arenas y Hermitaño Atencio, 2021; Romaní, 2020) o se comparan con otros países (Limaymanta et al., 2020; Armijos Valdivieso, Avolio Alecchi y Arévalo-Avecillas, 2021). En cambio, las investigaciones de estudios métricos de la información de alcance altmétrico a partir de publicaciones científicas de procedencia peruana son escasas o de reciente interés (Vílchez-Román, Huamán-Delgado y Alhuay-Quispe, 2021).

Mugnaini et al. (2021) afirman que los estudios sobre la disponibilidad y uso de los DOI (siglas en inglés de Digital Object Identifier) pueden ayudar en el flujo de información científica en la era digital y garantizar una mayor coherencia al realizar análisis bibliométricos. En ese sentido, las herramientas de monitoreo de la actividad altmétrica de las publicaciones basan la recolección de datos a partir de varios identificadores persistentes. Estos códigos de identificación varían de acuerdo con el tipo de documento, tipo de plataforma web o áreas de publicación, tales como:

a) Identificadores de repositorios: utilizados en plataformas del mismo nombre.

- PubMed iD en ciencias de la salud.

- arXiv iD en física, matemáticas y ciencias de la computación.

- ADS iD en astrofísica.

- SSRN iD en ciencias sociales. 
- RePEC iD en economía.

b) Identificadores por tipos de objetos:

- Handle usados en repositorios digitales.

- URN (Uniform Resource Name).

- URL: cualquier URL como de los repositorios.

- ISBN: usado en libros.

- YouTube iD: URL de video en YouTube.

- Clinical Trial iD: usado en repositorio de ensayos clínicos.

- OCLC iD: usado para registros del catálogo OCLC.

- DOI: utilizado para artículos, tesis, libros, archivos de datos, entre otros tipos de documentos.

Actualmente existen diferentes plataformas que rastrean datos altmétricos (Altmetric, ImpactStory, PaperCritic, PLoS Impact, Plum Analytics, Dimensions, ScienceCard, CrowdoMeter, NewsFlow), siendo Altmetric (altmetric. com) la plataforma comercial y proveedor de datos altmétricos más importante. Esta plataforma captura información del impacto de un documento de varias fuentes de medios sociales y calcula una puntuación ponderada denominada Altmetric Score (Robinson-García et al., 2014). Actualmente, incluye como fuente de datos a varias plataformas sociales en línea (Tabla 1).

\begin{tabular}{|c|c|c|c|}
\hline Dimensión & Alcance & Fuente & Situación \\
\hline Policy documents & $\begin{array}{c}\text { Documento de política, orientación } \\
\text { o directrices de una organización } \\
\text { gubernamental } \\
\text { o no gubernamental. }\end{array}$ & Sitios web oficiales & Vigente \\
\hline Mainstream media & $\begin{array}{c}\text { Colección de fuentes de noticias } \\
\text { curada manualmente y captadas } \\
\text { en tiempo real. }\end{array}$ & Más de 5000 medios & Vigente \\
\hline Blogs & $\begin{array}{l}\text { Seguimiento de una lista selec- } \\
\text { cionada de blogs académicos y no } \\
\text { académicos realizado automática- } \\
\text { mente mediante canales RSS. }\end{array}$ & Más de 15000 blogs & Vigente \\
\hline \multirow{2}{*}{$\begin{array}{c}\text { Online reference } \\
\text { managers }\end{array}$} & \multirow{2}{*}{$\begin{array}{c}\text { Menciones en recursos de } \\
\text { marcadores sociales y gestores } \\
\text { bibliográficos. }\end{array}$} & Mendeley & Vigente \\
\hline & & CiteULike & Descontinuada \\
\hline \multirow{2}{*}{$\begin{array}{c}\text { Post-publication } \\
\text { peer-review }\end{array}$} & \multirow{2}{*}{$\begin{array}{l}\text { Seguimiento en plataformas web } \\
\text { de reconocimiento de revisión de } \\
\text { expertos. }\end{array}$} & Publons & Vigente \\
\hline & & PubPeer & Descontinuada \\
\hline
\end{tabular}




\begin{tabular}{|c|c|c|c|}
\hline \multirow{6}{*}{ Social media } & \multirow{6}{*}{$\begin{array}{l}\text { Menciones en posts, grupos o } \\
\text { páginas públicas de varias fuentes } \\
\text { de redes sociales y microblogging. }\end{array}$} & Twitter & Vigente \\
\hline & & Facebook & Vigente \\
\hline & & Reddit & \multirow{4}{*}{ Descontinuada } \\
\hline & & Google+ & \\
\hline & & Pinterest & \\
\hline & & Linkedln & \\
\hline Patent citations & $\begin{array}{c}\text { Seguimiento de las citas de } \\
\text { patentes de varias jurisdicciones } \\
\text { de todo el mundo. }\end{array}$ & 10 oficinas de patentes & Vigente \\
\hline \multirow[b]{2}{*}{ Citations } & \multirow{2}{*}{$\begin{array}{l}\text { Citaciones recibidas } \\
\text { en documentos incluidos } \\
\text { en fuentes y bases de datos } \\
\text { de citación. }\end{array}$} & Dimensions & \multirow{2}{*}{$\begin{array}{l}\text { Vigente vía } \\
\text { Altmetric } \\
\text { Explorer }\end{array}$} \\
\hline & & Web of Science & \\
\hline \multirow{5}{*}{ Other online sources } & \multirow{5}{*}{$\begin{array}{l}\text { Seguimiento de atención de } \\
\text { varias fuentes no incluidas en las } \\
\text { categorías anteriores }\end{array}$} & Wikipedia & Vigente \\
\hline & & Q\&A Forums & Vigente \\
\hline & & Faculty Opinions & Vigente \\
\hline & & YouTube & Vigente \\
\hline & & Open Syllabus & Vigente \\
\hline
\end{tabular}

Tabla 1. Categorías de repercusión y fuentes de cobertura en Altmetric

Fuente: Altmetric (2020)

En líneas generales, los objetivos del artículo se enmarcan en responder las siguientes interrogantes:

- ¿En qué tipos de acceso se publican los artículos de cinco áreas temáticas de las Ciencias Sociales?

- ¿Cuál es la distribución de fuentes altmétricas según el tipo de acceso publicado?

- ¿Existe asociación entre las menciones obtenidas de las fuentes altmétricas?

- ¿Existe diferencia en la repercusión mediática según el tipo de acceso?

\section{Metodología}

\section{Diseño}

La investigación es de tipo descriptivo y correlacional, de carácter transversal y emplea la revisión documental a fin de identificar literatura previa sobre indicadores altmétricos. 


\section{Muestra}

Se emplea una muestra de tipo intencional no probabilística conformada por los artículos con filiación a instituciones peruanas indizados en Scopus para el periodo 2011-2020 y clasificados en cinco áreas de las Ciencias Sociales: Artes y Humanidades, Negocios y Contabilidad, Ciencias de la Decisión, Economía y Finanzas, y Psicología. A partir de una muestra inicial de 3092 artículos, se identifica una muestra final de 1186 artículos correspondientes a las publicaciones con identificador DOI y al menos una atención altmétrica recibida.

La recolección de los datos se realizó en abril de 2021 y se empleó a Scopus como fuente de datos para recuperar artículos científicos. Se construyó una estrategia de búsqueda que permitió identificar publicaciones de tipo "artículo" y de tipo "revisión" con filiación peruana (campo AFFILCOUNTRY) para los años de estudio y delimitado a cinco áreas temáticas dentro de las Ciencias Sociales del sistema de clasificación ASJC (All Science Journal Classification Codes) que emplea Scopus (Tabla 2).

El año de inicio para nuestro corte de tiempo (2011) corresponde al año después de la publicación del Manifiesto Altmetrics; se toma el umbral de tiempo a dos periodos de cinco años en referencia a los fundamentos de los indicadores basados en este periodo: H5-index, Impact factor 5-year.

Los datos exportados de Scopus en formato de archivo delimitado por comas (csv) fueron depurados, y luego se seleccionaron los artículos con identificador DOI para la recuperación de los datos altmétricos mediante el recurso Altmetric Explorer de la plataforma comercial Altmetric. La evidencia del tipo de acceso abierto a los documentos proviene de la información registrada en Dimensions y extraída desde Altmetric Explorer.

\section{Análisis de datos}

Los análisis estadísticos se efectuaron mediante el software IBM SPSS 23. En el análisis correlacional bivariado se emplea el coeficiente Rho de Spearman y la prueba de hipótesis considera valores $p$ inferiores a 0.05 como significativos. Los análisis incluyen las siguientes variables:

- Áreas: cinco áreas de ciencias sociales (Tabla 2).

- Documentos: número de artículos con DOI y actividad altmétrica.

- Menciones en Twitter.

- Menciones en Facebook.

- Repercusión en blogs.

- Repercusión en sitios de noticias. 
- Menciones en Wikipedia.

- Lecturas en Mendeley.

- Tipo de acceso: abierto (bronze, gold, green, hybrid) y restringido.

- Ratio mediática: cociente entre menciones en una fuente y documentos del área.

\begin{tabular}{|c|c|c|}
\hline Código & Categoría & Área ASJC \\
\hline \multirow[t]{14}{*}{ arts } & \multirow{14}{*}{$\begin{array}{l}\text { Arts } \\
\text { and Humanities }\end{array}$} & Archeology (arts and humanities) \\
\hline & & Arts and Humanities (miscellaneous) \\
\hline & & Arts and Humanities (all) \\
\hline & & Classics \\
\hline & & Conservation \\
\hline & & History \\
\hline & & History and Philosophy of Science \\
\hline & & Language and Linguistics \\
\hline & & Literature and Literary Theory \\
\hline & & Museology \\
\hline & & Music \\
\hline & & Philosophy \\
\hline & & Religious studies \\
\hline & & Visual Arts and Performing Arts \\
\hline \multirow[t]{11}{*}{ busi } & \multirow{11}{*}{$\begin{array}{c}\text { Business, } \\
\text { Management, } \\
\text { and Accounting }\end{array}$} & Business, Management, and Accounting (all) \\
\hline & & Accounting \\
\hline & & Business and International Management \\
\hline & & $\begin{array}{l}\text { Business, Management, and Accounting } \\
\text { (miscellaneous) }\end{array}$ \\
\hline & & Industrial relations \\
\hline & & Management Informational Systems \\
\hline & & Management of Technology and Innovation \\
\hline & & Marketing \\
\hline & & $\begin{array}{l}\text { Organizational Behavior and Human Resource } \\
\text { Management }\end{array}$ \\
\hline & & Strategy and Management \\
\hline & & Tourism, Leisure, and Hospitality Management \\
\hline
\end{tabular}




\begin{tabular}{|c|c|c|}
\hline \multirow[t]{5}{*}{ deci } & \multirow{5}{*}{$\begin{array}{l}\text { Decision } \\
\text { Sciences }\end{array}$} & Decision Sciences (all) \\
\hline & & Decision Sciences (miscellaneous) \\
\hline & & Information Systems and Management \\
\hline & & $\begin{array}{l}\text { Management Science and Operations } \\
\text { Research }\end{array}$ \\
\hline & & Statistics, Probability and Uncertainity \\
\hline \multirow[t]{4}{*}{ econ } & \multirow{4}{*}{$\begin{array}{l}\text { Economics, } \\
\text { Econometrics } \\
\text { and Finance }\end{array}$} & Economics, Econometrics, and Finance (all) \\
\hline & & $\begin{array}{l}\text { Economics, Econometrics, and Finance } \\
\text { (miscellaneous) }\end{array}$ \\
\hline & & Economics and Econometrics \\
\hline & & Finance \\
\hline \multirow[t]{8}{*}{ psyc } & \multirow[t]{8}{*}{ Psychology } & Applied Psychology \\
\hline & & Clinical Psychology \\
\hline & & Developmental and Educational Psychology \\
\hline & & Experimental and Cognitive Psychology \\
\hline & & Neuropsychology and Physiological Psychology \\
\hline & & Psychology (all) \\
\hline & & Psychology (miscellaneous) \\
\hline & & Social Psychology \\
\hline
\end{tabular}

Tabla 2. Áreas temáticas de ciencias sociales ASJC

\section{Resultados}

\section{Presencia en fuentes altmétricas}

La Tabla 3 muestra que de la población inicial recuperada (3 092 artículos), la cantidad de artículos con DOI representa 80 \% (2 466), mientras que sólo $38 \%$ son artículos con identificador DOI y por lo menos una actividad altmétrica que corresponde al número final de publicaciones incluidas en el análisis (1 186).

\begin{tabular}{|l|r|r|r|r|r|r|}
\hline \multicolumn{1}{|c|}{ Áreas } & \multicolumn{1}{c|}{ Docs } & \multicolumn{1}{c|}{ \% Docs } & \multicolumn{1}{c|}{ D0I } & \multicolumn{1}{c|}{ \% D0I } & \multicolumn{1}{c|}{$\begin{array}{c}\text { D0I }+ \\
\text { Altm }\end{array}$} & $\begin{array}{c}\text { \% D0I + } \\
\text { Altm }\end{array}$ \\
\hline arts & 872 & 28.20 & 661 & 26.80 & 290 & 24.45 \\
\hline busi & 788 & 25.49 & 585 & 23.72 & 230 & 19.39 \\
\hline deci & 198 & 6.40 & 131 & 5.31 & 49 & 4.13 \\
\hline econ & 639 & 20.67 & 538 & 21.82 & 289 & 24.37 \\
\hline
\end{tabular}




\begin{tabular}{|l|r|r|r|r|r|r|}
\hline psyc & 595 & 19.24 & 551 & 22.34 & 328 & 27.66 \\
\hline Total & 3092 & 100.00 & 2466 & 100.00 & 1186 & 100.00 \\
\hline
\end{tabular}

Docs: Total de documentos recuperados

DOI: Documentos recuperados con DOI

Altm: Documentos con Dol y repercusión altmétrica

Tabla 3. Proporción muestral de documentos con atención altmétrica

Los datos del análisis descriptivo (Tabla 4) muestran que Mendeley es la fuente que proporciona mayor número de menciones en fuentes altmétricas con una media de 49.63 pero también es la que mayor varianza en los datos (4 799.41) posee. Otras fuentes con mayor número de datos son las menciones en Twitter y las citas en Dimensions; mientras que los blogs y Wikipedia son las fuentes con menor datos altmétricos.

\begin{tabular}{|l|r|r|r|r|r|r|r|}
\hline \multicolumn{1}{|c|}{ Estadístico } & Blogs & News & Facebook & Twitter & Mendeley & Wikipedia & Dimensions \\
\hline Media & 0.11 & 0.22 & 0.22 & 5.64 & 49.63 & 0.06 & 11.84 \\
\hline Mediana & 0.00 & 0.00 & 0.00 & 1.00 & 27.00 & 0.00 & 4.00 \\
\hline Varianza & 0.34 & 2.36 & 0.41 & 354.60 & 4799.41 & 0.15 & 810.46 \\
\hline Desv. estándar & 0.58 & 1.54 & 0.64 & 18.83 & 69.28 & 0.39 & 28.47 \\
\hline Mínimo & 0.00 & 0.00 & 0.00 & 0.00 & 0.00 & 0.00 & 0.00 \\
\hline Máximo & 8.00 & 33.00 & 7.00 & 355.00 & 960.00 & 8.00 & 589.00 \\
\hline Rango & 8.00 & 33.00 & 7.00 & 355.00 & 960.00 & 8.00 & 589.00 \\
\hline Asimetría & 8.71 & 13.13 & 4.40 & 10.29 & 4.44 & 11.89 & 10.23 \\
\hline Curtosis & 93.97 & 222.56 & 27.32 & 144.34 & 36.23 & 199.04 & 165.06 \\
\hline
\end{tabular}

Tabla 4. Análisis descriptivo de las variables

La Tabla 5 presenta los datos acumulados de las menciones para las cinco áreas de estudio según las siete fuentes altmétricas. Los datos son favorables para las fuentes Mendeley y Twitter, para los campos de Economía (econ) y Psicología (psyc), que son dos de las tres áreas con mayor número de artículos analizados (size depending), mientras que las Ciencias de la Decisión (deci) presentan una menor puntuación altmétrica.

\begin{tabular}{|l|r|r|r|r|r|r|r|}
\hline Áreas & \multicolumn{1}{|c|}{ Blogs } & News & Facebook & Twitter & Mendeley & Wikipedia & Dimensions \\
\hline arts & 20 & 40 & 75 & 977 & 7.292 & 30 & 1.969 \\
\hline busi & 5 & 16 & 28 & 730 & 11.645 & 6 & 2.043 \\
\hline deci & 10 & 13 & 10 & 402 & 2.600 & 2 & 688 \\
\hline
\end{tabular}




\begin{tabular}{|l|r|r|r|r|r|r|r|}
\hline econ & 46 & 62 & 69 & 2.469 & 16.216 & 10 & 3.825 \\
\hline psyc & 46 & 125 & 84 & 2.101 & 21.055 & 27 & 5.511 \\
\hline
\end{tabular}

Tabla 5. Menciones altmétricas acumuladas por áreas

\section{Áreas de publicación y tipo de acceso}

El $54 \%$ de los artículos de Negocios y Contabilidad (125 de 230) analizados fueron publicados en revistas de acceso restringido, mientras que los manuscritos de Psicología (203 de 328) y de Ciencias de la Decisión (30 de 49 artículos) fueron publicados en acceso abierto con $62 \%$ y $61 \%$ respectivamente del total de artículos revisados (Figura 1).

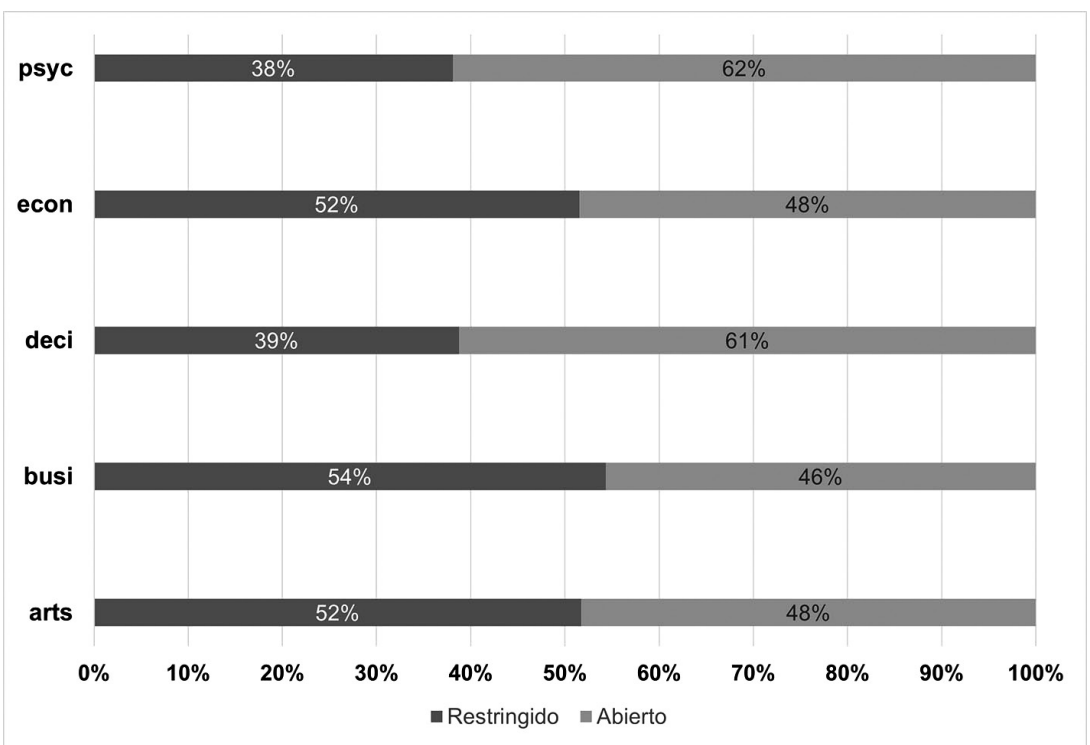

Figura 1. Proporción del tipo de acceso según área

Un análisis desagregado por tipo de acceso abierto y fuentes de datos altmétricos, empleando un cociente entre la cantidad de menciones sobre el número de artículos del área denominado "ratio mediática", muestra que las menciones en Mendeley y las citas en Dimensions predominan en las rutas de acceso restringido y verde; por otro lado, la proporción mediática en Twitter aparece representada en casi todas las rutas de publicación (Figura 2). 


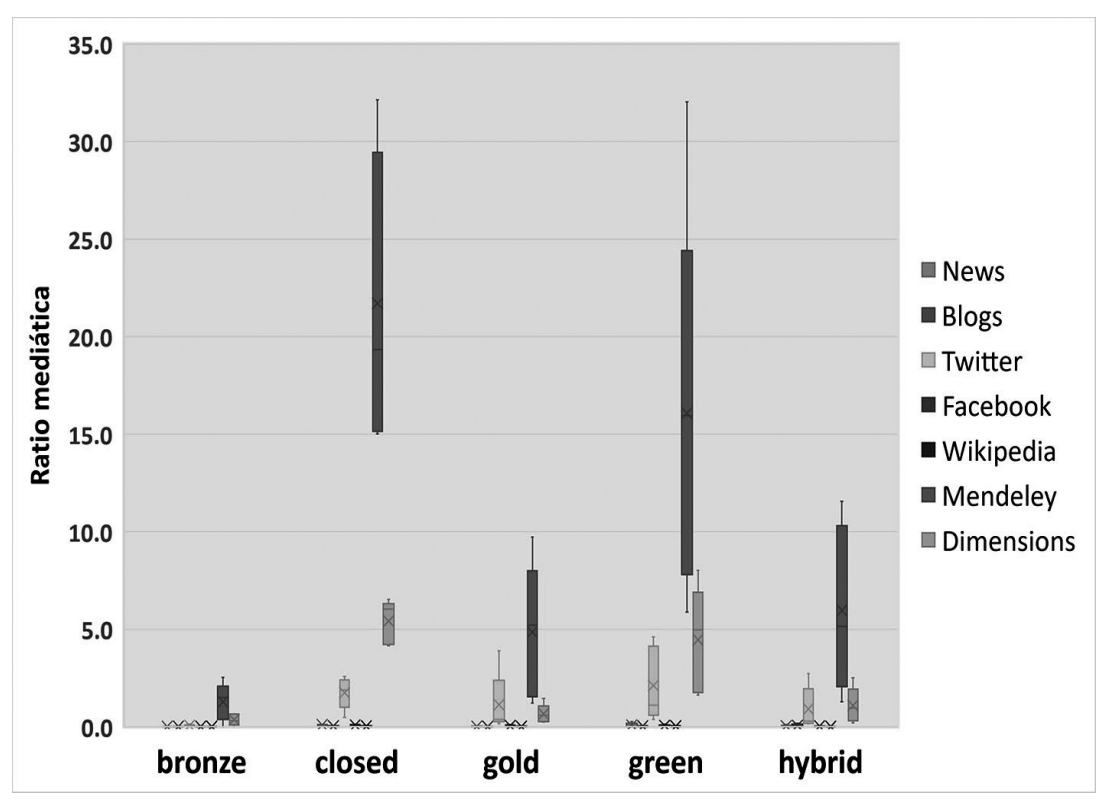

Figura 2. Ratio de menciones por documento según fuentes y tipo de acceso

\section{Correlación de indicadores}

Se obtuvo que existe una alta correlación entre el número de citas en Dimensions y Mendeley $(r=0.797)$, además de una asociación moderada $(r=0.413)$ entre las menciones en blogs y sitios de noticias; mientras que para los demás indicadores altmétricos la correlación resulta muy baja (Tabla 6).

\begin{tabular}{|l|c|c|c|c|c|c|c|}
\hline Altmétricas & $\mathbf{1}$ & $\mathbf{2}$ & $\mathbf{3}$ & $\mathbf{4}$ & $\mathbf{5}$ & $\mathbf{6}$ & $\mathbf{7}$ \\
\hline 1. Blog & 1.000 & 0.413 & 0.141 & 0.159 & 0.184 & 0.105 & 0.164 \\
\hline 2. News & & 1.000 & 0.155 & 0.172 & 0.180 & 0.111 & 0.188 \\
\hline 3. Facebook & & & 1.000 & 0.127 & 0.137 & 0.047 & 0.102 \\
\hline 4. Twitter & & & & 1.000 & 0.246 & 0.057 & 0.134 \\
\hline 5. Mendeley & & & & & 1.000 & 0.114 & 0.797 \\
\hline 6. Wikipedia & & & & & & 1.000 & 0.147 \\
\hline 7. Dimensions & & & & & & & 1.000 \\
\hline
\end{tabular}

Nota: La correlación es significativa en el nivel 0.01 (bilateral)

Tabla 6. Análisis de correlación entre indicadores altmétricos 
La Figura 3 presenta la distribución de los datos del análisis bivariado con mayor asociación a partir de la Tabla 6, se muestra un análisis complementario mediante el coeficiente de determinación lineal obteniéndose una alta correlación $\left(\mathrm{R}^{2}=0.650\right)$.

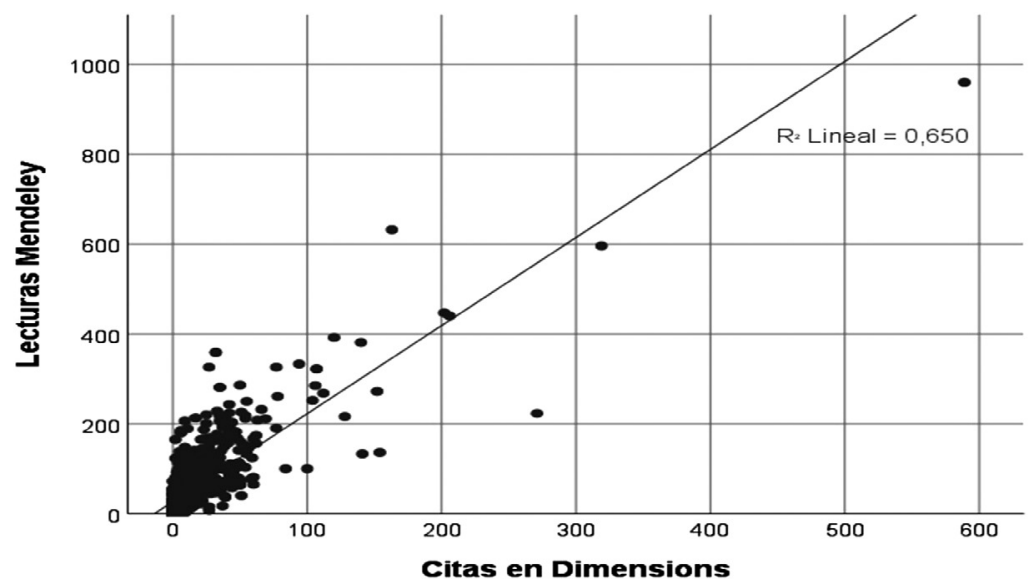

Figura 3. Correlación entre citas de Dimensions y lecturas de Mendeley

Finalmente, como respuesta a la última pregunta del estudio, se evidenció que existe diferencia estadísticamente significativa según el tipo de acceso (abierto o restringido) sólo para las menciones provenientes de Facebook ( $p=.000)$ y Twitter $(p=.005)$, pero no para la repercusión altmétrica obtenida de las demás fuentes (Tabla 7).

\begin{tabular}{|l|c|c|c|c|c|c|c|}
\hline \multicolumn{1}{|c|}{ Estadístico } & Blog & News & Facebook & Twitter & Mendeley & Wikipedia & Dimensions \\
\hline $\begin{array}{l}\text { U de Mann- } \\
\text { Whitney }\end{array}$ & 176446.0 & 175911.0 & 189459.5 & 191383.0 & 169349.0 & 175751.0 & 163927.0 \\
\hline $\begin{array}{l}\text { W de } \\
\text { Wilcoxon }\end{array}$ & 367099.0 & 366564.0 & 380112.5 & 382036.0 & 360002.0 & 366404.0 & 354580.0 \\
\hline $\begin{array}{l}\text { Estadístico } \\
\text { de contraste }\end{array}$ & 176446.0 & 175911.0 & 189459.5 & 191383.0 & 169349.0 & 175751.0 & 163927.0 \\
\hline $\begin{array}{l}\text { Sig. Asintó- } \\
\text { tica* }\end{array}$ & 0.615 & 0.772 & 0.000 & 0.005 & 0.318 & 0.802 & 0.053 \\
\hline
\end{tabular}

* Bilateral. Nivel de significancia $=.05$

\section{Tabla 7. Estadísticos de contraste por tipo de acceso}




\section{Discusión}

Estudios previos analizaron la correlación entre indicadores bibliométricos (número de citas recibidas) e indicadores altmétricos (capturas, menciones y uso), como Thelwall et al. (2013) y Costas, Zahedi y Wouters (2015), que analizan si las altmetrics se complementan y en qué medida con los indicadores tradicionales, incluso contemplan las diferencias disciplinares. De igual forma, algunos estudios tratan sobre el impacto de las métricas alternativas en áreas temáticas específicas, como Htoo y $\mathrm{Na}$ (2017), que revisan el impacto altmétrico de artículos incluidos en la colección SSCI (Science Citation Index) de Web of Science y quienes encontraron que la repercusión en medios altmétricos favorece a las publicaciones de tres áreas biomédicas que a las sociales (Library Science, Business-Finance, Law, Political Science). Por su parte, Mohammadi y Thelwall (2014) estudiaron artículos de las categorías WoS con temáticas en Ciencias Sociales y Humanidades, y evidenciaron que existe mayor correlación entre estadísticas de lecturas y citas en tres de las 10 áreas analizadas: Business \& Economics $(p=0.573)$, Information Science \& Library Science ( $p=0.535)$, y Psychology ( $p=0.514)$.

La mayoría de los estudios previos estudian la proporción de acceso abierto de los artículos extraídos de Web of Science, Scopus y otros recursos de literatura científica, pero son escasos los estudios a partir de publicaciones procedentes de un país o región. Piwowar et al. (2018) reportan que más de $50 \%$ de las publicaciones en investigación biomédica y matemáticas son de libre acceso, mientras que cerca de $80 \%$ de los artículos en química e ingeniería se encuentran en acceso cerrado. Martín-Martín et al. (2018) evidencian que $5 \%$ de los artículos de ciencias sociales y del comportamiento se encuentran disponibles por la vía bronce de acceso abierto y $14 \%$ en la ruta verde. Singh, Piryani y Srichandan (2020) reportan que las publicaciones de India en las rutas verde y dorada suman $33 \%$ en las áreas de Ciencias Sociales. Martín-Martín et al. (2018) reportan que estudios previos identifican la disponibilidad de acceso abierto de los artículos a partir de diferentes fuentes como Unpaywall, DOAJ, ROAR, OpenDOAR, ResearchGate, Google Académico o hasta el propio buscador de Google, por lo que una limitante de la investigación se enmarca en la disponibilidad de las rutas de acceso abierto proporcionadas por la propia base de datos Scopus.

En las Ciencias de la Información (y Bibliotecología), algunos autores demuestran que la preferencia por la publicación en acceso abierto permite que los artículos obtengan un mayor impacto científico (citas) y en las social media (lecturas) (Cintra, Furnival y Milanez, 2017). Otros concluyen que la gestión de contenidos (metadatos) y el uso de un sistema de gestión de revistas (OJS) 
contribuyen a incrementar el uso o consumo de los artículos (vistas, descargas) y eventualmente, repercutirá en el incremento del impacto científico de la revista y los artículos publicados (Uribe Tirado, Vallejo Echavarría y Betancur Marín, 2016).

En América Latina, las iniciativas regionales de acceso abierto tienen larga trayectoria y son ampliamente reconocidas por los países de la región y también a nivel global. Por un lado, se encuentra el proyecto SciELO Network (www.scielo.org), que se clasifica como vía de publicación en ruta dorada, según la definición de Piwowar et al. (2018); por el otro lado, iniciativas como La Referencia (www.lareferencia.info), que consiste en una red regional de repositorios institucionales mayormente provenientes de universidades de varios países latinoamericanos, permiten la explotación de la ruta verde. En el caso del Perú, hasta el primer semestre de 2020 se reportan en DOAJ 92 revistas en acceso abierto y el recolector nacional ALICIA agrega 169 repositorios institucionales (Concytec, 2021). Sin embargo, pese a la considerable cantidad de repositorios peruanos, la proporción de artículos frente a los demás tipos de documentos depositados en los repositorios de universidades del sector privado apenas se acerca a $6 \%$ y en las universidades públicas a 0.1 \% (Alhuay-Quispe et al., 2017). Por lo que, aunque en los países en los que la infraestructura de acceso abierto es relativamente buena, es necesario desarrollar una infraestructura de acceso abierto mediante la cooperación (Seo, 2018).

Finalmente, ya sea que el artículo se publique en acceso abierto o que el autor asuma el APC ante la revista para la apertura del manuscrito, y que el comité editorial de una revista gestione de manera óptima las publicaciones, la presencia de los propios autores en las diversas plataformas sociales y científicas, y más aún, la constante interacción conducirán a obtener mayor visibilidad e impacto de su productividad desde lo altmétrico a lo bibliométrico (Uribe-Tirado y Alhuay-Quispe, 2017). Asimismo, los beneficios del acceso abierto deben comprenderse junto con las dificultades inherentes para gestionar este nuevo enfoque de la publicación académica para beneficio de la sociedad (Ellers, Crowther y Harvey, 2017).

\section{Conclusiones}

- Los autores peruanos de Negocios y Contabilidad publican mayormente en revistas de acceso restringido; en cambio, en el área de Psicología publican generalmente en revistas bajo alguna ruta de acceso abierto. 
- Las publicaciones peruanas en Ciencias Sociales obtienen mayor visibilidad en las fuentes de Mendeley y Dimensions siempre y cuando los artículos se publiquen por la ruta verde de acceso abierto e incluso en acceso restringido.

- Las lecturas obtenidas en Mendeley guardan estrecha relación con las citas recibidas en Dimensions, mientras que las menciones en blogs y sitios de noticias muestran una ligera asociación.

- La repercusión mediática en artículos peruanos de Ciencias Sociales se diferencia por el tipo de acceso únicamente en las fuentes de Facebook y Twitter.

Agradecimientos

Nuestros agradecimientos al "Programa de acceso a los datos para investigadores" de Altmetric (https://www.altmetric.com/research-access/) a través del cual se logró obtener los datos altmétricos para el presente estudio.

\section{REFERENCIAS}

Alhuay-Quispe, Joel y Lourdes Bautista-Ynofuente. 2020. "Imprecisión de los datos en estudios altmétricos". Revista Cubana de Información en Ciencias de la Salud 31 (4): e1655.

http://www.rcics.sld.cu/index.php/acimed/article/view/1655

Alhuay-Quispe, Joel, David Quispe-Riveros, Lourdes Bautista-Ynofuente y Josmel Pacheco-Mendoza. 2017. "Metadata Quality and Academic Visibility Associated with Document Type Coverage in Institutional Repositories of Peruvian Universities". Journal of Web Librarianship 11 (3-4): 241-254.

https://doi.org/10.1080/19322909.2017.1382427

Alonso-Arévalo, Julio. 2016. "Altmetrics pueden no seguir siendo alternativa por mucho tiempo". Revista ORL 7 (3): 169-177.

https://doi.org/10.14201/orl201673.14190

Altmetric. 2020. "Sources of Attention". https://www.altmetric.com/about-our-data/our-sources/

Armijos Valdivieso, Pablo, Beatrice Avolio Alecchi y Danny Arévalo-Avecillas. 2021. "Factors That Influence the Individual Research Output of University Professors: The Case of Ecuador, Peru, and Colombia". Journal of Hispanic Higher Education. OnlineFirst. https://doi.org/10.1177/15381927211008684

Beasley, Gerald. 2016. "Article Processing Charges: A New Route to Open Access?” Information Services and Use 36 (3-4): 163-170. https://doi.org/10.3233/ISU-160815 
Bornmann, Lutz. 2014. "Do altmetrics point to the broader impact of research? An overview of benefits and disadvantages of altmetrics". Journal of Informetrics 8 (4): 895-903.

https://doi.org/10.1016/j.joi.2014.09.005

Carranza Esteban, Renzo F., Oscar Mamani-Benito, Josué R. Turpo Chaparro, Percy G. Ruiz Mamani y Ronald M. Hernández. 2020. "Student Scientific Output in Social Science Journals: A review of Peru, Ecuador and Paraguay”. International Journal of Early Childhood Special Education 12 (1): 425-432. https://doi.org/10.9756/INT-JECSE/V12I1.201022

Cintra, Paulo Roberto, Ariadne Chloe Furnival y Douglas Henrique Milanez. 2017. "Vantagens de citação do acesso aberto em periódicos selecionados da Ciência da Informação: uma análise ampliada aos indicadores altmétricos”. Informação EInformação 22 (1): 129-149. https://doi.org/10.5433/1981-8920.2017v22n1p129

Concytec (Consejo Nacional de ciencia, Tecnología e Innovación Tecnológica). 2021. "Listado de instituciones y repositorios participantes". Fecha de consulta: 9 de junio de 2021. https://alicia.concytec.gob.pe/instituciones/

Costas, Rodrigo, Zohreh Zahedi y Paul Wouters. 2015. "Do altmetrics correlate with citations? Extensive comparison of altmetric indicators with citations from a multidisciplinary perspective". Journal of the Association for Information Science and Technology 66 (10): 2003-2019. https://doi.org/10.1002/asi.23309

Delgado Arenas, Raúl, Antonio L. Delgado Arenas y Bernardo C. Hermitaño Atencio. 2021. "Determinantes para publicación de artículos científicos en revistas indexadas: caso Universidad Nacional Agraria del Perú”. Revista General de Información y Documentación 31 (1): 317-330. https://doi.org/10.5209/rgid.76972

Ellers, Jacintha, Thomas W. Crowther y Jeffrey A. Harvey. 2017. "Gold Open Access Publishing in Mega-Journals: Developing Countries Pay the Price of Western Premium Academic Output”. Journal of Scholarly Publishing 49 (1): 89-102. https://doi.org/10.3138/jsp.49.1.89

Harnad, Stevan. 2005. "The Implementation of the Berlin Declaration on Open Access: Report on the Berlin 3 Meeting Held 28 February - 1 March 2005, Southampton, UK". D-Lib Magazine 11 (3).

https://doi.org/10.1045/march2005-harnad

Haustein, Stefanie. 2016. "Grand challenges in altmetrics: Heterogeneity, data quality and dependencies". Scientometrics 108 (1): 413-423.

https://doi.org/10.1007/s11192-016-1910-9

Haustein, S., T. D. Bowman y R. Costas. 2015. "When is an article actually published? an analysis of online availability, publication, and indexation dates", en Proceedings of ISSI 2015 Istanbul: 15th International Society of Scientometrics and Informetrics Conference, 1170-1179. https://www.issi-society.org/proceedings/issi_2015/1170.pdf

Htoo, Tint Hla Hla y Jin-Cheon Na. 2017. "Disciplinary differences in altmetrics for social sciences”. Online Information Review 41 (2): 235-251. https://doi.org/10.1108/OIR-12-2015-0386 
Lahikainen, Johanna. 2016. "Altmetrics in Social Sciences and Humanities: Possibilities, Challenges, and Experiences”. Trabajo presentado en IFLA World Library and Information Congress - WLIC 2016, Columbus, OH - Connections. Collaboration. Community, Ohio, USA, 16 de agosto de 2016.

http://library.ifla.org/id/eprint/1356

Limaymanta, César H., Hilda Zulueta-Rafael, Cristina Restrepo-Arango y Patricio Alvarez-Muñoz. 2020. "Análisis bibliométrico y cienciométrico de la producción científica de Perú y Ecuador desde Web of Science (2009-2018)”. Información, Cultura y Sociedad (43): 31-52.

https://doi.org/10.34096/ics.i43.7926

Livia, José. 2008. "La producción científica y los estudios de post grado en Psicología en el Perú”. Interamerican Journal of Psychology 42 (3): 431-445.

Martín-Martín, Alberto, Rodrigo Costas, Thed Van Leeuwen y Emilio Delgado López-Cózar. 2018. "Evidence of Open Access of Scientific Publications in Google Scholar: A Large-Scale Analysis”. Journal of Informetrics 12 (3): 819-841. https://doi.org/10.1016/j.joi.2018.06.012

Melero, Remedios. 2015. "Altmetrics - a complement to conventional metrics". Biochemia medica 25 (2): 152-160. https://doi.org/10.11613/BM.2015.016

Mohammadi, Ehsan y Mike Thelwall. 2014. "Mendeley readership altmetrics for the social sciences and humanities: Research evaluation and knowledge flows". Journal of the Association for Information Science and Technology 65 (8): 1627-1638. https://doi.org/10.1002/asi.23071

Mugnaini, Rogério, Grischa Fraumann, Esteban F. Tuesta y Abel L. Packer. 2021. "Openness trends in Brazilian citation data: factors related to the use of DOIs". Scientometrics 126:2523-2556. https://doi.org/10.1007/s11192-020-03663-7

Peña-Rey Lorenzo, Isabel. 2004. "Estudio bibliométrico de la producción científica sobre dioxinas a través de las bases de datos Pubmed e I.M.E. (19972003)”. Tesis doctoral, Universidad de Murcia, España. http://hdl.handle.net/10201/167

Piwowar, Heather, Jason Priem, Vincent Larivière, Juan Pablo Alperin, Lisa Matthias, Bree Norlander, Ashley Farley, Jevin West y Stefanie Haustein. 2018. "The State of OA: A Large-Scale Analysis of the Prevalence and Impact of Open Access Articles". Peerj 6: e4375. https://doi.org/10.7717/peerj.4375

Robinson-García, Nicolas, Daniel Torres-Salinas, Zohreh Zahedi y Rodrigo Costas. 2014. "New data, new possibilities: Exploring the insides of altmetric.com". El profesional de la información 23 (4): 1386-6710. https://doi.org/10.3145/epi.2014.jul.03

Romaní, Franco. 2020. "Análisis bibliométrico de las publicaciones científicas originales del Instituto Nacional de Salud del Perú en el periodo 1998-2018”. Revista Peruana de Medicina Experimental y Salud Pública 37 (3): 485-494. https://doi.org/10.17843/rpmesp.2020.373.5470

Seo, Tae-Sul. 2018. "Open Access Full-Text Databases in Asian Countries". Science Editing 5 (1): 26-31. https://doi.org/10.6087/kcse.114 
Singh, Vivek Kumar, Rajesh Piryani y Satya Swarup Srichandan. 2020. "The Case of Significant Variations in gold-green and Black Open Access: Evidence from Indian Research Output”. Scientometrics 124 (1): 515-531. https://doi.org/10.1007/s11192-020-03472-y

Sud, P. y M. Thelwall. 2014. "Evaluating altmetrics”. Scientometrics 98, 1131-1143. https://doi.org/10.1007/s11192-013-1117-2

Thelwall, Mike, Stefanie Haustein, Vincent Larivière y Cassidy R. Sugimoto. 2013. "Do altmetrics work? Twitter and ten other social web services”. PloS one 8 (5): e64841. https://doi.org/10.1371/journal.pone.0064841

Uribe-Tirado, Alejandro y Joel Alhuay-Quispe. 2017. "Estudio métrico de ALFIN en Iberoamérica: de la bibliometría a las altmetrics". Revista española de Documentación Científica 40 (3): e180. https://doi.org/10.3989/redc.2017.3.1414

Uribe Tirado, Alejandro, Juan Camilo Vallejo Echavarría y Darío Alexander Betancur Marín. 2016. "Somos visibles y tenemos impacto. Análisis desde datos de acceso abierto, altmetrics y otros de la Revista Interamericana de Bibliotecología”. Revista Interamericana de Bibliotecología 39 (3): 243-275. https://doi.org/10.17533/udea.rib.v39n3a04

Vílchez-Román, Carlos, Farita Huamán-Delgado y Joel Alhuay-Quispe. 2021. "Social dimension activates the usage and academic impact of Open Access publications in Andean countries: a structural modeling-based approach". Information Development 37 (2): 209-220. https://doi.org/10.1177/0266666920901849

Wilsdon, James, Liz Allen, Eleanora Belfiore, Philip Campbell, Stephen Curry, Steven Hill, Richard Jones, Roger Kain, Kerridge Simon, Mike Thelwall, Jane Tinkler, Ian Viney, Paul Wouters, Jude Hill y Ben Johnson. 2015. The metric tide: Report of the independent review of the role of metrics in research assessment and management. HEFCE.

https://kar.kent.ac.uk/81123/

Para citar este texto:

Alhuay-Quispe, Joel y Lourdes Bautista-Ynofuente. 2021. "Repercusión altmétrica y tipo de acceso en artículos peruanos de Ciencias Sociales”. Investigación Bibliotecológica: archivonomía, bibliotecología e información 35 (89): 91-110.

http://dx.doi.org/10.22201/iibi.24488321xe.2021.89.58369 